\title{
Lymphatic Muscle Cells in Rat Mesenteric Lymphatic Vessels of Various Ages
}

\author{
Eric A. Bridenbaugh, PhD, Irina Tsoy Nizamutdinova, MD, PhD, Daniel Jupiter, PhD, ${ }^{1}$ \\ Takashi Nagai, MD, $\mathrm{PhD}^{1,3}$ Sangeetha Thangaswamy, $\mathrm{PhD}$, \\ Victor Chatterjee, MBBS, and Anatoliy A. Gashev, MD, PhD, D Med Sci ${ }^{1}$
}

\begin{abstract}
Background: Recent studies on aging-associated changes in mesenteric lymph flow in situ demonstrated predominance of the severe negative chronotropic effect of aging on the contractility of aged mesenteric lymphatic vessels (MLV). At the same time, contraction amplitude of the aged vessels was only slightly diminished by aging and can be rapidly stimulated within 5-15 minutes. However, the detailed quantitative evaluation of potential aging-associated changes in muscle cells investiture in MLV has never been performed.

Methods and Results: In this study we, for the first time, performed detailed evaluation of muscle cells investiture in MLV in reference to the position of lymphatic valve in different zones of lymphangion within various age groups (3-mo, 9-mo and 24-mo Fischer-344 rats). Using visual and quantitative analyses of the images of MLV immunohistochemically labeled for actin, we confirmed that the zones located close upstream (pre-valve zones) and above lymphatic valves (valve zones) possess the lowest investiture of lymphatic muscle cells. Most of the high muscle cells investiture zones exist downstream to the lymphatic valve (post-valve zones). The muscle cells investiture of these zones is not affected by aging, while pre-valve and valve zones demonstrate significant aging-associated decrease in muscle cells investiture.

Conclusions: The low muscle cells investiture zones in lymphatic vessels consist of predominantly longitudinally oriented muscle cells which are positioned in pre-valve and valve zones and connect adjacent lymphangions. These cells may provide important functional impact on the biomechanics of the lymphatic valve gating and electrical coupling between lymphangions, while their aging-associated changes may delimit adaptive reserves of aged lymphatic vessels.
\end{abstract}

\section{Introduction}

A GING TARGETS MULTIPLE BODY SYSTEMS including the lymphatic system, which is tightly involved in the maintenance of fluid and macromolecule homeostasis and immune cell trafficking in all other organ systems of the body. Despite some advancements in aging-related research in last decades, there is still limited information available on the aging-associated changes in structure and function of the lymphatic system, and particularly lymphatic vessels. Observations on the age-related alterations in structure of lymphatic vessels are mostly limited by observations of such changes in the largest lymphatic trunk of the body-thoracic duct, with some limited information about other regional lymphatic networks. Zhdanov ${ }^{1,2}$ and Zerbino ${ }^{3}$ reported re- sults of their observations on aging-induced changes in morphology of various human lymphatic networks in the early 1960s. They mentioned the general tendency of the reduction of number of lymphatic capillaries (nonmuscular initial lymphatics) through all of the body. These authors also noted presence of specific "varicose bulges," which exist in muscular lymphatic vessels in various lymphatic networks. The number of such bulges increases with age. Aged thoracic duct has defined signs of lipid accumulation, thickening, and fibrosis. Rabinovitz and Saphir in 1965 also reported signs of fibrosis, wall thickening, and lipid droplet accumulation in the wall of aged human thoracic duct, while consistent agingassociated changes in muscle cells of the thoracic duct wall have not been mentioned. These findings were re-confirmed later. ${ }^{4}$ Another study was published more than 2 decades ago

\footnotetext{
${ }^{1}$ Department of Medical Physiology, College of Medicine, Texas A \& M Health Science Center, Temple, Texas.

${ }^{2}$ Department of Surgery, Texas A \& M Health Science Center/Scott \& White Healthcare, Temple, Texas.

${ }^{3}$ Department of Physiology, Shinshu University School of Medicine, Matsumoto, Japan.
} 
on the morphological structure of the vessel walls of various lymphatic vessels in elderly humans. ${ }^{5}$ The authors showed ${ }^{5}$ that in the aged human, the destruction of the elastic elements and atrophy of muscle cells in the thoracic duct wall resulted in the development of "duct sclerosis". Investigations of the human mesenteric lymphatic bed demonstrated that after age of 65, the number of collecting lymphatic vessels in the human mesentery was significantly reduced, and the number of connections between lymphatic vessels of the same lymphatic arcade level was greatly diminished. Moreover, the authors observed considerable signs of degradation of the blood capillaries in the lymphatic wall after the age of 60 . In some preparations of collecting lymphatic vessels, aneurysm-like formations containing only endothelial cells in their walls were found, primarily in the areas located downstream but close to the base of lymphatic valves. However, these authors did not perform more detailed investigation of aged mesenteric lymphatic networks, and did not mention any observations of the aging-associated changes in muscle cells investiture in mesenteric lymphatic vessels (MLV). Recently, reduction of nerve supply was also reported for the aged human thoracic duct and other lymphatic vessels. ${ }^{6,7}$ In conclusion, the aging-associated changes in structure of the regional lymphatic networks have not been systematically studied, especially with links to the functional status of the aged lymphatic networks.

Our recently published preliminary observations on agingassociated changes in orientation and investiture of muscle cells in mesenteric lymphatic vessels ${ }^{8}$ revealed some signs of decreased number and changes in orientation of mesenteric lymphatic muscle cells. However, such studies in the past were never performed in detail. In this study, we, for the first time, performed immunohistochemical labeling and imaging of smooth muscle actin in mesenteric lymphatic vessels isolated from animals of three age groups (3-mo: representing juvenile; 9-mo: representing adulthood, and 24-mo: representing senility) in order to perform quantitative evaluation of potential aging-associated changes in muscle cells investiture in MLV over these selected ages with reference to the zones with lymphatic valves.

\section{Materials and Methods}

\section{Animals and surgery}

For the current studies, 3-, 9- and 24-mo-old male Fischer344 (F-344) rats were obtained from the aged rat colony maintained by the NIH National Institute of Aging. This is a commonly used rat strain in aging-related research, ${ }^{9,10}$ and the age groups selected represent juvenile, adulthood, and senility, respectively. All animal procedures for current studies were reviewed and approved by our Institutional Animal Care and Use Committee.

\section{Isolation of mesenteric lymphatic vessels}

To isolate mesenteric lymphatic vessels, rats were anesthetized with Nembutal Sodium Solution ( $50 \mathrm{mg} / \mathrm{kg}$ IP). A 4-cm long midline abdominal incision was made through the skin, underlying fascia, and muscle layers. A small loop of intestine $6-7 \mathrm{~cm}$ in length was exteriorized through the incision. A section of mesentery containing lymphatic vessels was positioned in a custom dissection chamber within the field of view of a stereomicroscope and continuously suffused with phosphate buffered saline (PBS) (EMD Chemicals, Gibbstown, NJ, USA, catalog \# 6505). Sections of MLV 1-3 cm in length were carefully dissected and excised from the animal. After isolation of the MLV, the rats were euthanized with Nembutal Sodium Solution (120 mg/kg body weight IC).

\section{Immunohistochemical labeling of MLV for CD31, smooth muscle actin, and MHC class I/ proteins}

Isolated 3-mo, 9-mo, and 24-mo-old Fischer-344 rat MLV were cannulated on glass pipettes in a custom-made single vessel chamber containing PBS and then flushed using PBS perfusion. The vessels were then fixed for $30 \mathrm{~min}$ at $0^{\circ} \mathrm{C}$ in $100 \%$ methanol and washed three times for $5 \mathrm{~min}$ each with $0.05 \%$ Tween-20 in phosphate-buffered saline (PBST). The vessels were blocked overnight at $4{ }^{\circ} \mathrm{C}$ in $5 \%$ normal goat serum (Jackson ImmunoResearch Laboratories, Inc., West Grove, PA, USA, catalog \# 005-000-121) in PBST, washed three times for $15 \mathrm{~min}$ each in PBST, and then incubated $1 \mathrm{~h}$ in PBST containing mouse monoclonal antibodies directed against CD31 (platelet endothelial cell adhesion molecule, PECAM-1) to visualize endothelial cell junctions; clone TLD-3A12, IgG1 isotype, BD Pharmingen/BD Biosciences, San Jose, CA, USA, catalog \# 555025; dilution 1:250), smooth muscle actin to visualize muscle cells; clone CGA7, IgG2b isotype, Santa Cruz Biotechnologies, Santa Cruz, CA, USA, catalog \# sc-53015; dilution 1:200), and MHC class II (major histocompatibility complex II) to visualize antigen presenting cells; clone 10-3.6, IgG2a isotype, BioLegend; San Diego, CA, USA, catalog \# 109902; dilution 1:250. After washing three times for $15 \mathrm{~min}$ each with PBST, the vessels were incubated $1 \mathrm{~h}$ in PBST containing Alexa Fluor 488 goat anti-mouse IgG1 (catalog \# A21370, dilution 1:200), Alexa Fluor 555 goat anti-mouse IgG2b (catalog \# A21435, dilution 1:200), and Alexa Fluor 647 goat anti-mouse IgG2a (catalog \# A31573, dilution 1:200), (all: Life Technologies, Grand Island, NY, USA). After further washing in PBST, the vessels were mounted on glass slides under \#1.5 coverslips using ProLong Gold antifade reagent (Life Technologies, catalog \# P36934).

\section{Epifluorescence imaging and actin fluorescence intensity analysis}

The fixed and fluorescently-labeled MLV were imaged using an Olympus CKX41 fluorescent microscope equipped with a Lumen 200 Fluorescence Illumination System and filter sets suitable for Alexa Fluor 488, 555, and 647 imaging. Images were captured using an Olympus LUCPLFLN 20X objective (0.45 N.A.) and an Olympus DP72 camera controlled by Olympus CellSens digital imaging software. Care was taken to center the lymphatic valves within each image, and vessel images that exhibited evidence of damage or poor immunohistochemical labeling were excluded from subsequent analyses.

To quantify the lymphatic muscle cells investiture via fluorescence immunohistochemistry across the lymphatic valve region and to assess the changes in muscle cells investiture across the aging process, we used a smooth muscle cellspecific monoclonal antibody directed against smooth muscle actin (clone CGA7) predominantly presented in rat MLV. ${ }^{11}$ In preliminary studies, we found no evidence of changes in all actin isoforms within MLV across the aging process via 
Western blot (data not shown), and thus, densitometric analysis of actin labeling was considered a reliable indicator of muscle cells investiture in this experimental system. as well as can be used as reference protein for densitometric analysis of Western blot experimental data on various proteins obtained from animals of different ages. We believe that analyzing total intensity of fluorescence of actin gave a good indication of the "total volume" of the muscle cells in lymphatic wall (muscle cell investiture), while we were not able to differentiate precisely variations in volumes/sizes of the single muscle cells and to define their exact number per tissue volume to calculate muscle cells density.

To assess muscle cells investiture across the MLV valve area, the valve images were imported into NIH ImageJ software, and the actin fluorescence intensity (AFI) was measured before, within, and after the valve region within each image using the CD31 image to delineate the valve location. Within each age group (i.e., 3-mo, 9-mo, and 24-mo), we carried out measurements of AFI expressed in absorption units (AU) to compare the muscle cells investiture in these three zones of the lymphangions: pre-valve, valve, and post-valve through determination of the mean AFI for each zone within each imaged segment of MLV. For image analysis, we used only those images of MLV that have consistent levels of signal along all vessel segments and absence of any defects in staining. Because these criteria are somewhat subjective, we used the following approach for analysis of AFI. For subset \#1 of images, the investigator, who performed all epifluorescent imaging as described in Methods, selected acceptable images himself and used the following criteria to define the zones within lymphangions: only images of MLV segments with valve position close to the middle of image were used. For each vessel, the perimeter of the lymphatic endothelial cell image was traced from the CD31 staining. Then two lines were drawn approximately perpendicular to the vessel wall and positioned one at the downstream tip of the valve leaflets and the other at the furthest upstream point on base of the valve leaflets. These two lines divided the perimeter tracing of the vessel wall into three zones with all lymphatic endothelial cells associated with the valve being located in the central "valve" region. The other ends of the pre-valve and post-valve zones were limited by edges of the image. Then these three zone tracings were transferred to the muscle cell image and the densitometry was performed. Subset \# 1 contains 20 MLV images from four 3-mo-old rats, 15 MLV images from four 9mo-old rats, and $27 \mathrm{MLV}$ images from four 24-mo-old rats. To double check the consistency of results, for subset \#2 of images, another person independently selected images from the same pool of images as used for subset \# 1, having lymphatic valves located within image so that both pre-valve and postvalve zones have been presented within single image. The following criteria have been used to define the zones within lymphangions: valve zone defined from the downstream tip of the valve leaflets to the furthest upstream point on base of the valve leaflets. Portions of the pre-valve zones located immediately upstream to the lymphatic valve and equal to the length of the given lymphatic valve or limited by edges of the image if latter is lesser, and portions of post-valve zones located downstream to the lymphatic valve (after the ends of valve leaflets) and limited by edges of the image were used for analysis. The AFI was analyzed via densitometry using the NIH ImageJ program. Multiple, equal-sized regions of inter- est (ROI) were selected within each image. Mean ROI intensities were averaged separately for across the image within pre-valve, valve, and post-valve zones of lymphangion for each image of MLV segment. Subset \# 2 contains $25 \mathrm{MLV}$ images from four 3-mo-old rats, 23 MLV images from four 9mo-old rats, and $20 \mathrm{MLV}$ images from four 24-mo-old rats. Finally, for subset \# 3 we pooled together images of MLV obtained from 9-mo and 24-mo-old rats that have been obtained for various other projects but had CD31/actin staining performed as described above. Another person selected acceptable images and performed analysis by the same way as used for subset \# 2. Subset \# 3 contains 75 MLV images from 31 9-mo-old rats, and 73 MLV images from 27 24-mo-old rats.

To be able to compare data between all three subsets of images, we used relative ratios of AFI within each analyzed image. After we discovered from our visual observations and confirmed by analysis of fluorescence of images (see Results and Fig. 2A) that AFI (and accordingly muscle cells investiture) is highest in post-valve zone, we, within each image used for analysis, accepted the mean AFI for this zone as 100\% and calculated ratios for pre-valve and valve zones by dividing their mean AFIs by AFI of the corresponding post-valve zone. Subsequently for all subsets we determined averages for pre-valve and valve zones' ratios within each age group.

Statistical differences were determined for each subset of images separately by two-way ANOVA, regression analysis and paired Student's $t$-test (JMP software version 9.0.0. for Windows) as appropriate and considered significant at $p \leq 0.05$.

\section{Results}

\section{Visual observations of the muscle cells investiture in various zones of the lymphangions}

Figure 1 demonstrates the representative epifluorescent images of the MLV from the same age groups that were selected from images used for analysis of subset \#1. We found that, in the majority of cases, muscle cells investiture is relatively consistent in the long regions downstream to lymphatic valves on rat MLV (partially these zones shown on Fig. 1). These "high muscle cells investiture" zones within MLV, which by our estimation take $92 \%-95 \%$ of the vessel length, stay to a large degree morphologically intact in aged vessels. (However, with selected resolution of images we were not able to demonstrate on Fig. 1 these zones in full length without losing quality of images, especially in pre-valve and valve zones).

As consistent finding we repeatedly observed in walls of MLV in all age groups the regions with discontinuous and irregular muscle cells organization, which look like "pseudo-holes" on solely actin-stained images (Fig. 1, orange staining). These low muscle cells investiture zones located immediately upstream to the lymphatic valve of the same vessels (pre-valve zone) and often-near valve (valve zone). Our visual observations did not reveal any consistent differences in the shape and size of these "low muscle cells investiture" zones between 3-mo and 9-mo-old MLV. However, we paid attention that these "low muscle cells investiture" zones occurred to be larger in 24-mo-old MLV; subsequent analysis confirmed these observations (see below). Interestingly, the "low muscle cells investiture" zones do not appear to be associated with antigen presenting cells, resident population of 

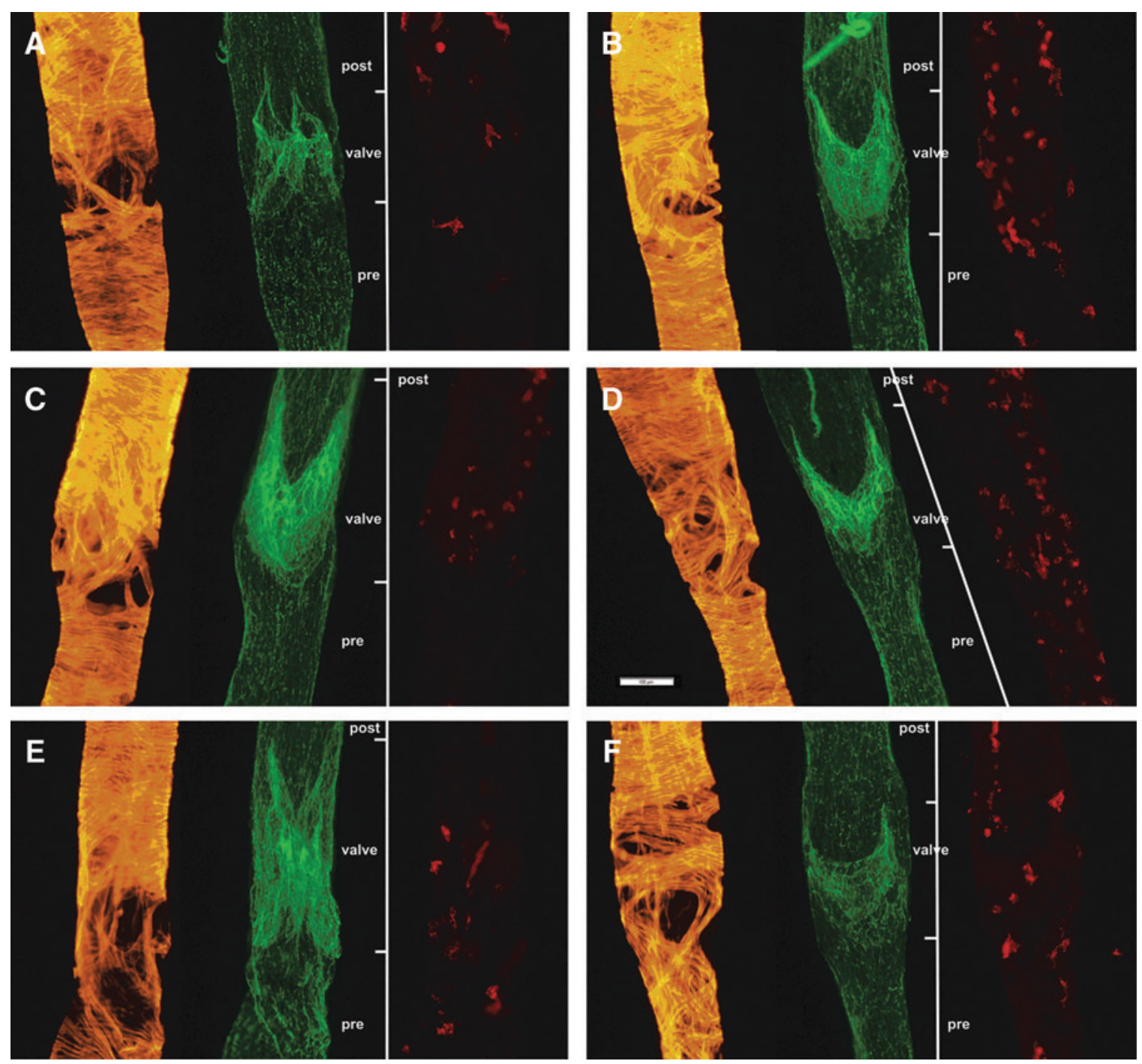

FIG. 1. Representative epifluorescent images of rat MLV isolated from 3-mo (A, B), 9-mo (C, D), and 24-mo (E, F) Fischer344 rats. Smooth muscle actin is stained in orange to indicate lymphatic muscle cells. CD31 is stained in green to indicate lymphatic endothelial cell junctions and therefore to delineate location of lymphatic valves. Lines located right to each image labeled by CD31 demonstrate locations of pre-valve (pre), valve, and post-valve (post) zones as they were defined on these images for subsequent analysis. Major histocompatibility complex II is stained in red to indicate antigen presenting cells. Scale bar represents $100 \mu \mathrm{m}$ and applies to all images.

which was demonstrated for rat MLV by Zawieja group ${ }^{12,13}$ (Fig. 1, red staining). During work on this project we were not able to reveal considerable aging-associated changes in number of these cells in walls of rat MLV.

\section{Quantitative analysis of the muscle cells investiture in various zones of the lymphangions}

To assess whether the muscle cells investiture of the prevalve and valve zones was indeed significantly different than in post-valve zone, we performed a measurements of the intensity of fluorescent signal generated by immunohistochemically labeled actin, AFI, across the three zones of lymphangion (pre-valve, valve, and post-valve) in MLV within each age group. We found that in all age groups, the post-valve zone remained aging-intact, while the pre-valve and valve zones exhibited significantly less muscle cell investiture compared to the post valve zone (Fig. 2A). Moreover, we found no significant differences in muscle cells investiture between pre-valve and valve zones between 3-mo and 9-mo-old MLV, while the same zones in 24-mo-old MLV experienced decrease in muscle cells investiture. Pre-valve zone in 24-mo-old MLV had 21\% less fluorescent signal for actin versus 9-mo-old MLV, valve zone had $23 \%$ less for the 24-mo versus 9-mo-old pair. As we explained in Methods, these data for levels of fluorescent signal were obtained from subset \# 1 of MLV images, generated in the same conditions and processed by the same investigator. We concluded that for all ages the muscle cells investiture in post-valve zone remains the same, and can be used for relative comparisons of AFI with other zones, removing therefore any potential concerns about variability in actin-generated fluorescent signal 

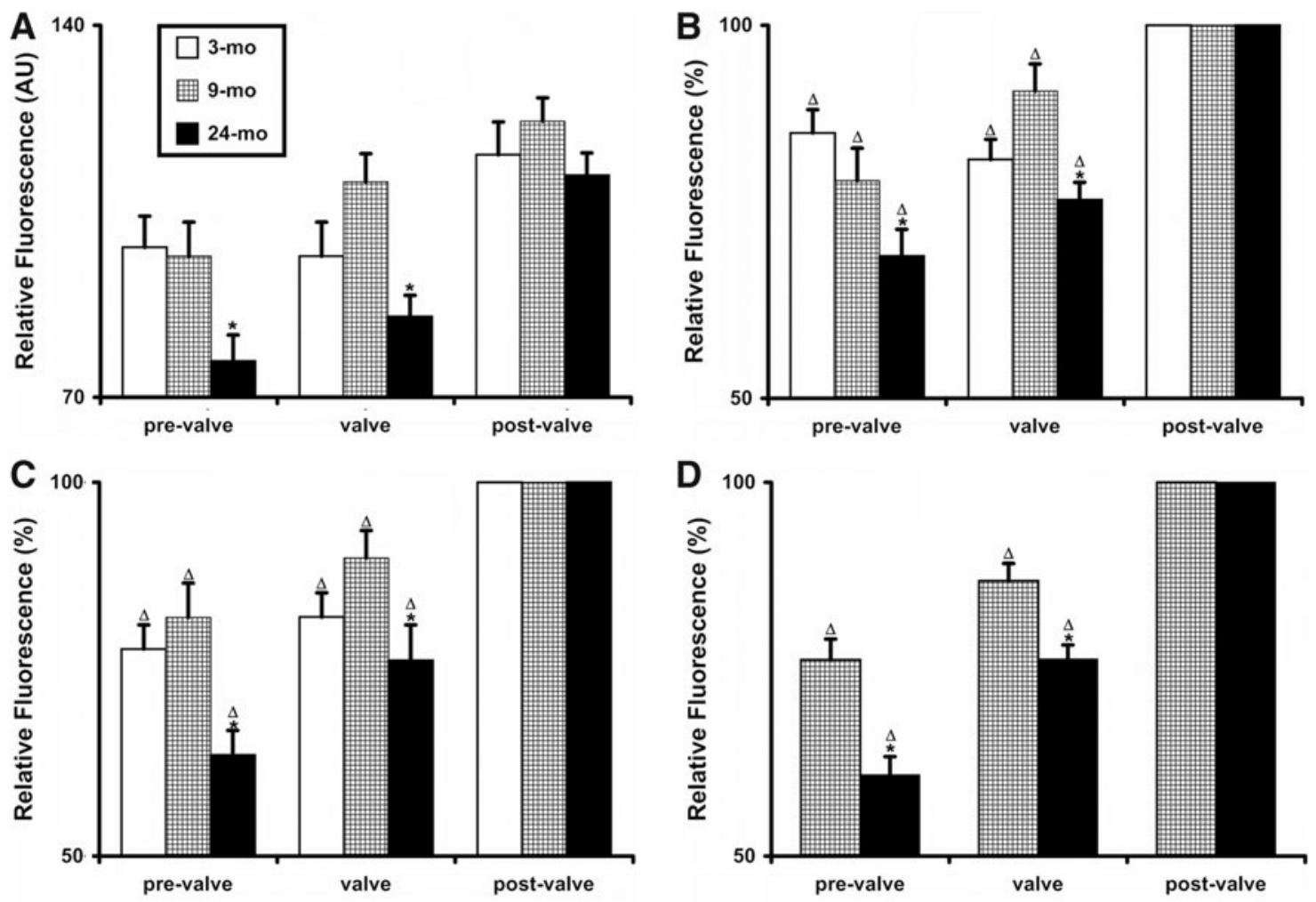

Zone of the Lymphangion

Zone of the Lymphangion

FIG. 2. Quantitative analysis of relative fluorescence (muscle cells investiture) in young (3-mo), adult (9-mo), and aged (24mo) segments of rat mesenteric lymphatic vessels. (A) Relative fluorescence measured in AU for subset \# 1 (see Methods); (B-D) relative fluorescence ratios determined for subsets \# 1, 2, and 3, correspondingly. Values are means \pm SEM; ${ }^{*}$ indicates significant differences $(p<0.05)$ between 9 -mo and 24 -mo age groups; $\Delta$ indicates significant differences in relative fluorescence ratios between pre-valve, valve, and post-valve zones in lymphangion within corresponding age group.

between MLV segments processed in different days (subsets \# 1 and \# 2), and furthermore, between MLV processed by different investigators in different types of experiments in different days for subset \# 3 . As a result we continued our quantitative analysis of the muscle cells investiture for all subsets of images using the ratio of fluorescence "pre-valve/ post-valve" and "valve/post-valve" expressed in percentage of post-valve AFI.

Figure 2, panels B, C, and D presents these "normalized to post-valve zone" results for subsets 1,2 , and 3, correspondingly. We found that results obtained from different subsets of images demonstrated the same order of values, and same trends and same statistical significances as within as between all selected age groups (compare panels B, C, and D on Figure 2). Cumulatively, between all three subsets in 3-mo-old MLV pre-valve zone had $16 \%-22 \%$ less muscle cells investiture than post-valve zone, valve zone had $18 \%$ less muscle cells investiture than post-valve zone. In 9-mo-old MLV pre-valve zone had $19 \%-23 \%$ less muscle cells investiture than post-valve zone, valve zone had 9\%-13\% less muscle cells investiture than post-valve zone. For 24-mo-old MLV these numbers were: pre-valve zone had $26 \%-31 \%$ less muscle cells investiture than post-valve zone, valve zone had $24 \%$ less muscle cells investiture than post-valve zone. While comparing all averages obtained for these ratio between 9-mo and 24-mo-old MLV, we found that aging-associated decrease in muscle cells investiture in pre-valve and valve zones of the lymphangion was statistically significant in all analyzed subsets of images (Fig. 2B, C, and D) (compare these cumulative averages provided above for 9-mo versus 24-mo-old NMLV - pre-valve: $19 \%-23 \%$ versus $26 \%-31 \%$; valve: $9 \%-$ $13 \%$ versus $24 \%$ ).

\section{Observations of longitudinally-oriented muscle cells which cross-connect lymphangions over low muscle cells investiture zones}

The term "lymphangion" was first introduced by Mislin, ${ }^{14-16}$ and is considered to be the structural unit of the muscularized lymphatic vessel. It includes the lymphatic valve, post-valve zone, and downstream pre-valve zone all the way up to the next valve, that can contract independently, providing peristalsis-like propagation of the contractile wave along the lymphatic vessel. ${ }^{17,18}$ Using this commonly accepted nomenclature in this study we analyzed pre-valve zones of the previous upstream lymphangion and valve and post-valve zones of the next downstream lymphangion. As a regular finding through all analyzed specimens we observed that in all MLV the low muscle cells investiture zones consist mostly of longitudinally-oriented (along the direction of lymph flow) or close to longitudinallyoriented muscle cells, which as a single cell, or bundles of few cells together connect adjacent lymphangions (opposite 

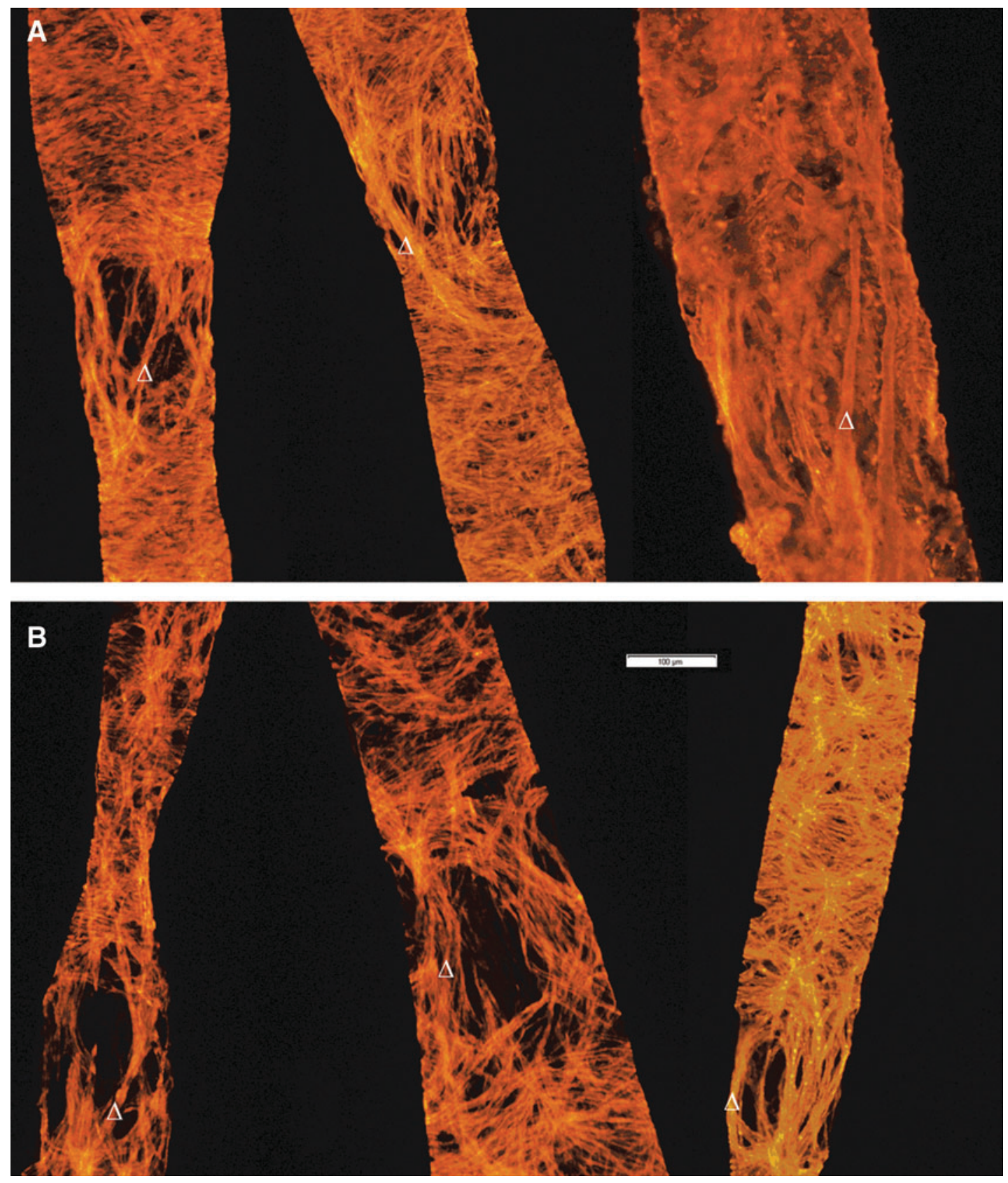

FIG. 3. Representative epifluorescent images of rat MLV isolated from 9-mo (A) and 24-mo (B) Fischer rats. Smooth muscle actin is stained in orange to indicate lymphatic muscle cells. Images demonstrate existence of the longitudinally oriented muscle cells that connect two adjacent lymphangions over of the zone with comparatively low investiture of the lymphatic muscle cells. At each presented image of MLV, $\Delta$ symbol points to example of the single longitudinally oriented muscle cell. Scale bar represents $100 \mu \mathrm{m}$ and applies to all images.

to predominantly circular or close to circular orientation of the majority of muscle cells in post-valve zones). We observed this phenomenon in MLV of different ages and did not find any visible differences in the shape of these cells related to age of the vessel. We believe that observed agingassociated changes in muscle cells investiture of pre-valve and valve zones are due to the decreased number of such cells: we were not able to count muscle cells using experimental approaches implemented in this study. Figure 3 provides representative images of 9-mo (Panel A) and 24- mo-old (Panel B) MLV with such cells existing in the low muscle cells investiture zones.

\section{Discussion}

The existence of low muscle cells investiture zones in lymphatic vessels has been observed by different lymphatic researchers; we may refer this to the recent personal communications with Drs. David Zawieja, Michael Davis, and Dirk van Helden. In this study, we for the first time performed 
the detailed evaluation of muscle cells investiture of various zones in rat mesenteric lymphatic vessels with reference to position of their valves within various ages representing young, adult, and aged animals. We found unique features of the distribution of lymphatic muscle cells in valvular zones of lymphatic vessels and determined their aging-associated alterations. We believe that these structural data have important functional implications, providing some ground for explanation of the previous physiological findings and for some potential extensions of studies on lymphatic function in future.

Our observations reveal that majority of circularly-oriented muscle cells located in post-valve zones of the lymphatic vessels constitute $92 \%-95 \%$ percent of total vessel length and we believe that muscle cells in these zones provide the greatest impact on development of the lymphatic contractile force. We did not find any significant aging-associated differences in the investiture of muscle cells on these post-valve zones. Such finding by our opinion explains the fact that aging-associated depletion of the lymphatic contractile amplitude is not as profound as aging-associated inhibition of the contraction frequency observed in aged mesenteric lymphatic vessels. ${ }^{19,20}$ Therefore, functionally, the aging-associated changes in lymphatic muscle cells appears to be less important for lymph pumping and lymph flow in mesenteric lymphatic network in resting conditions, but may have greater impact at the periods of greater functional demands to contracting lymphatic vessels that periodically exist in lymphatic networks during conditions of high lymph formation and flow. The greater degree of the aging-associated depletion of the lymphatic contractile amplitude at higher levels of transmural pressure strongly supports this idea. ${ }^{19,21}$

We also found that the low muscle cells investiture zones are predominantly located upstream (pre-valve zone) and above (valve zone) the lymphatic valves. The functional importance of this phenomenon still needs to be determined. In light of recent studies with characterization of permeability of rat mesenteric lymphatic vessels, ${ }^{22}$ we may propose that these low muscle cells investiture zones may have important if not greatest impact on this process. The fact that these zones are increased by size in aged animals may predispose the aged lymphatic vessels to increased permeability especially during inflammation-related alterations in lymphatic endothelial cells, the only one layer of which without muscle cell coverage separates lumen of lymphatic vessels from adventitia and then surrounding tissues in these zones. At the same time, molecular traffic in opposite direction may also occur easier in such zones of the lymphatic vessels. Similar assumptions may be extrapolated to traffic of various immune cells through lymphatic wall in such mostly thin areas of the lymphatic wall, as well as to spread of various lymph-borne pathogens and cancer cells. At the same time, our observations that the "low muscle cells investiture" zones do not appear to be associated with antigen presenting cells, may additionally confirm the recent findings ${ }^{12,13}$ that these cells consist at least in part of the population of true lymphatic wall residents which require much thicker portion of vessel wall for their stable permanent position within MLV rather than one endothelial cell layer-containing wall of low muscle cells investiture pre-valve and valve zones.

Two important functional implications belong to our demonstration that the low muscle cells investiture zones in lymphatic wall consist predominantly of longitudinally ori- ented muscle cells that connect the pre-valve zone of the previous upstream lymphangion and post-valve zone of the next downstream lymphangion. The shape and position of these muscle cells assume that during lymphatic contraction these cells will shorten longitudinally inducing displacement of two adjacent lymphangions to get their positions during lymphatic systole closer to each other. Additionally, since these muscle cells are connected with structures in close proximity to the base of lymphatic valve leaflets, during lymphatic systole these muscle cells may introduce the active component of lymphatic valve closure, working like "lymphatic valve reins". At the same time these muscle cells may serve as structural substrate of bi-directional electrical coupling between two adjacent lymphangions, providing ground for bi-directional propagation of lymphatic contractile waves. ${ }^{18,23}$ Both of these functions are likely altered with aging since there is decrease in investiture of such longitudinally oriented muscle cells in the pre-valve and valve zones of the aged lymphangion. As we already mentioned above, these zones consist mostly of longitudinally-oriented or close to longitudinally-oriented muscle cells, and these zones are mostly affected by aging. In reference to the biomechanics of the lymphatic valve, we may propose that limited ability of these "lymphatic valve reins" to provide their impact on lymphatic valve closure may negatively influence contractility of aged lymphatic vessels. Recent findings demonstrated complexity of the lymphatic contractile behavior and high ability of the contracting lymphangions to adapt their pumping to the different levels of variable lymphatic preloads and afterloads. ${ }^{24-26}$ Such adaptation cannot exist without an effective lymphatic valve gating. ${ }^{27}$ Therefore we propose that in elderly the decrease of accessory muscle elements surrounding lymphatic valve may limit the ability of aged lymphatic vessels to adequately adapt their contractility to various preload/afterload challenges through disrupted mechanisms of the lymphatic valve gating. Such may create predisposition to the lowering of lymphatic productivity and to the increase of the fraction of reversed flow ${ }^{28}$ in aged vessels with subsequent formation of lymph stasis and potential spread of pathogens and immune cells in direction opposite to the direction of the normal lymph flow. Finally, we believe that the thin-walled low muscle cells investiture zones in aged MLV may finally be transformed to aneurysm-like formations, which were called as "varicose bulges" in old literature (see Introduction). Such formations are ideal places for formation of low-velocity turbulent lymph flow and accumulation of various molecules, pathogens, and cancer cells, which may later cross the thin, endothelial cell layer wall of these abnormal formations. Finally we believe that further investigations are necessary to confirm our current conclusions and to answer important questions related to findings of the current study.

In conclusion, we for the first time, performed detailed evaluation of muscle cells investiture in mesenteric lymphatic vessels in reference to the position of lymphatic valve in different zones of lymphangion within various age groups. Using visual and quantitative analyses of the images of the lymphatic vessels immunohistochemically labeled for actin, we confirmed that their pre-valve and valve zones possess the lowest investiture of lymphatic muscle cells. Most of the high muscle cells investiture zones exist downstream to the lymphatic valve (post-valve zone). The muscle cells investiture of these zones is not affected by aging, while pre-valve and valve zones 
demonstrate significant aging-associated decrease in muscle cells investiture. The low muscle cells investiture zones in lymphatic vessels consist of predominantly longitudinally oriented muscle cells which are positioned above and near lymphatic valve and connect adjacent lymphangions. These cells may provide important functional impact on the biomechanics of the lymphatic valve gating and electrical coupling between lymphangions, while their aging-associated changes may delimit adaptive reserves of aged lymphatic vessels.

\section{Acknowledgments}

The authors thank Kelci Jackson and Stacy Henderson for their help with image analysis.

\section{Author Disclosure Statement}

This work was supported in parts by the National Institutes of Health (NIH RO1 AG-030578 and HL-094269) and by Texas A\&M Health Science Center College of Medicine and Department of Medical Physiology. Drs. Bridenbaugh, Nizamutdinova, Jupiter, Nagai, Thangaswamy, and Gashev and Mr. Chatterjee have no conflicts of interest or financial ties to disclose.

\section{References}

1. Zhdanov DA. [Senile changes in the lymphatic capillaries and vessels]. Arkhiv anatomii, gistologii i embriologii, 1960;39:24-36.

2. Jdanov DA. On senile changes in lymphatic capillaries and vessels. J Cardiovasc Surg 1966;7:108-116.

3. Zerbino DD. [Senile changes in the outflow lymphatic vessels]. Arkhiv anatomii, gistologii i embriologii, 1960;39:3742.

4. Jozsef L, Laszlo M, Gabor L. [Changes in the structure of the wall of the human thoracic duct in relation to atherosclerosis and age]. Morphologiai es igazsagugyi orvosi szemle 1976; 16:43-47.

5. Orlov RS, Borisov AV, Borisova RP. Lymphatic vessels. Structure and mechanisms of contractile activity. (In Russian).1983, Leningrad, USSR: Nauka. 253 pages.

6. Mignini F, Sabbatini M, Cavallotti C. Analysis of nerve supply pattern in thoracic duct in young and elderly men. Lymphat Res Biol 2012;10:46-52.

7. Mignini F, Sabbatini M, Coppola L, Cavallotti C. Analysis of nerve supply pattern in human lymphatic vessels of young and old men. Lymphat Res Biol 2012;10:189-197.

8. Gashev AA. Basic mechanisms controlling lymph transport in the mesenteric lymphatic net. Ann NY Acad Sci 2010; 1207:E16-20.

9. Turturro A, Witt WW, Lewis S, Hass BS, Lipman RD, Hart RW. Growth curves and survival characteristics of the animals used in the Biomarkers of Aging Program. J Gerontol A Biol Sci Med Sci 1999;54: B492-501.

10. Nadon NL. Maintaining aged rodents for biogerontology research. Lab Anim (NY), 2004;33:36-41.

11. Muthuchamy M, Gashev A, Boswell N, Dawson N, Zawieja D. Molecular and functional analyses of the contractile apparatus in lymphatic muscle. FASEB J 2003;17:920-922.

12. Kuan IL, Bridenbaugh EA, Victora GD, et al. Surveillance of lymph by adipose macrophages localized in the wall of collecting lymphatic vessels. In: 9th World Congress for Microcirculation. 2010. Paris, France: International Scientific Committee of the Congress.
13. Wang W, Zawieja DC. A resident population of APC within the muscularized vessel walls of the rat lymphatic network. FASEB J 2011;25:632.-635.

14. Mislin H. Experimental detection of autochthonous automatism of lymph vessels. (In German). Experientia 1961;17:29-30.

15. Mislin H. Structural and functional relations of the mesenteric lymph vessels. New Trends in Basic Lymphology; Proceedings of a Symposium held at Charleroi (Belgium); July 11-13, 1966. Experientia 1966;14: 87-96.

16. Mislin H, Schipp R. Structural and functional relations of the lymph vessels. Progress in Lymphology; Proceedings of the International symposium on lymphology; Zurich, Switzerland, July 19-23, 1966.1966:360-365.

17. McHale NG, Roddie IC. Pumping activity in isolated segments of bovine mesenteric lymphatics. J Physiol 1975;244: 70P-72P.

18. McHale NG, Roddie IC. The effect of transmural pressure on pumping activity in isolated bovine lymphatic vessels. J Physiol 1976;261:255-269.

19. Nagai T, Bridenbaugh EA, Gashev AA. Aging-associated alterations in contractility of rat mesenteric lymphatic vessels. Microcirculation 2011;18:463-473.

20. Akl TJ, TNagai T, Cote GL, Gashev AA. Mesenteric lymph flow in adult and aged rats. Am J Physiol Heart Circ Physiol 2011;301:H1828-1840.

21. Gasheva OY, Knippa K, Nepiushchikh ZV, Muthuchamy M, Gashev AA. Age-related alterations of active pumping mechanisms in rat thoracic duct. Microcirculation 2007;14: 827-839.

22. Scallan JP, Huxley VH. In vivo determination of collecting lymphatic vessel permeability to albumin: A role for lymphatics in exchange. J Physiol 2010;588:243-254.

23. Zawieja DC, Davis KL, Schuster R, Hinds WM, Granger HJ. Distribution, propagation, and coordination of contractile activity in lymphatics. Am J Physiol 1993;264:H1283-1291.

24. Scallan JP, Wolpers JH, Muthuchamy M, Zawieja DC, Gashev AA, Davis MJ. Independent and interactive effects of preload and afterload on the pump function of the isolated lymphangion. Am J Physiol Heart Circ Physiol 2012;303: H809-824.

25. Davis MJ, Scallan JP, Wolpers JH, Muthuchamy M, Gashev AA, Zawieja DC. Intrinsic increase in lymphangion muscle contractility in response to elevated afterload. Am J Physiol Heart Circ Physiol 2012;303:H795-808.

26. Scallan JP, Wolpers JH, Davis MJ. Constriction of isolated collecting lymphatic vessels in response to acute increases in downstream pressure. J Physiol 2013;591:443-459.

27. Davis MJ, Rahbar E, Gashev AA, Zawieja DC, Moore, JE Jr. Determinants of valve gating in collecting lymphatic vessels from rat mesentery. Am J Physiol Heart Circ Physiol 2011; 301:H48-60.

28. Gashev AA. The mechanism of the formation of a reverse fluid filling in the lymphangions. (In Russian). Fiziologicheskii Zhurnal SSSR Imeni I. M. Sechenova, 1991;77: 3-69.

Address correspondence to: Anatoliy A. Gashev Department of Medical Physiology College of Medicine

Texas AEM Health Science Center 702 SW H.K. Dodgen Loop Temple, TX 76504

E-mail: gashev@tamu.edu 